\title{
O protagonismo do enfermeiro nas ações de educação em saúde na estratégia saúde da família
}

\author{
The nurse's protagonism in health education actions in the family health strategy \\ El papel del enfermero en las acciones de educación em salud en la estrategia de salud familiar
}

\begin{abstract}
RESUMO
Objetivo: Compreender a atuação e o protagonismo do enfermeiro nas ações de educação em saúde na Estratégia Saúde da Família (ESF). Método: Estudo descritivo de abordagem qualitativa. A Coleta dos dados foi realizada entre novembro de 2018 e abril de 2019, através de entrevistas semiestruturadas. A Amostra foi composta por 08 enfermeiros da ESF. Critérios de inclusão: ser enfermeiro da ESF, está presente no dia da coleta e assinar o TCLE. Resultados: A partir da análise emergiram três categorias: As rodas de conversas nas ações de educação em saúde; Entraves na execução das ações de educação em saúde; Lacuna na Educação Permanente dos enfermeiros da ESF. Conclusão: A educação em saúde é instrumento essencial para melhoria dos indicadores de saúde da população, através da mudança de comportamento do usuário. As ações de educação permanente são fundamentais para empoderar os enfermeiros nesse processo de participação do usuário no cuidado de sua saúde.
\end{abstract}

DESCRITORES: Enfermeiro; Educação em Saúde; Estratégia Saúde da Família

\section{ABSTRACT}

Objective: To comprehend on the performance and protagonism of nurses in health education activities in the Family Health Strategy (FHS). Method: Descriptive study with a qualitative approach. Data collection was conducted between November 2018 and April 2019, through semi-structured interviews. The sample was composed of 08 FHS nurses. Inclusion criteria: To be a nurse of the FHS, to be present on the day of collection and signing the ICF. Results: From the content analysis of the interviews three categories emerged: The conversation wheels in health education actions; Barriers in the execution of health education actions; Gap in the Permanent Education of FHS nurses. Conclusion: Health education is an essential tool for improving the health indicators of a population, by changing the user's behavior. The actions of continuing education are essential to empower nurses in this process of user participation in their health care.

DESCRIPTORS: Nurse; Health Education; Family Health Strategy.

\section{RESUMEN}

Objetivo: Comprender sobre el actuación y el protagonismo de la enfermera en las actividades de educación para la salud en la Estrategia de Salud Familiar (ESF). Método: Un estudio descriptivo con un enfoque cualitativo. Los datos se recogieron entre noviembre de 2018 y abril de 2019 mediante entrevistas semiestructuradas. La muestra estaba compuesta por 08 enfermeras del ESF. Criterios de inclusión: ser enfermera de la ESF, estar presente el día de la recogida y firmar el TCLI. Resultados: A partir del análisis del contenido de las entrevistas emergen tres categorías: Las vías de conversación en las acciones de educación en salud; Entraves en la ejecución de las acciones de educación en salud; Lacuna en la educación permanente de los enfermeros del ESF. Conclusión: La educación sanitaria es una herramienta esencial para mejorar los indicadores de salud de una población, mediante el cambio de comportamiento del usuario. Las acciones de formación continua son esenciales para capacitar a las enfermeras en este proceso de participación de los usuarios en sus cuidados sanitarios.

DESCRIPTORES: Enfermera; educación sanitária; estrategia de salud familiar.

RECEBIDO EM: 30/06/2021 APROVADO EM: 18/10/2021

\section{Yris Luana Rodrigues da Silva}

Enfermeira, graduada pela Universidade Estadual da Paraíba, docente efetiva da Escola Técnica Estadual Arlindo Ferreira dos Santos (Sertânia-PE). Mestre em Educação no Ensino da Área da Saúde (FPS) -Especialista em Saúde Pública (FIP).

ORCID: 0000-0003-2184-1151

\section{Juliana Monteiro Costa}

Psicóloga, Doutora em Psicologia Clínica pela Universidade Católica de Pernambuco. Docente da Graduação de Psicologia da 


\section{artigo}

Silva, Y. L. R., Costa, J. M.

O protagonismo do enfermeiro nas ações de educação em saúde na estratégia saúde da familia

Faculdade Pernambucana de Saúde (FPS). Docente Permanente do Mestrado Profissional em Educação para o Ensino na Área de Saúde da FPS e Docente Permanente do Mestrado Profissional em Psicologia da Saúde da FPS.

ORCID:0000-0002-6976-8670

\section{INTRODUÇÃO}

A criação do SUS (Sistema Único de Saúde) e o movimento de reforma pela saúde fortaleceram a Atenção Primária. A Política Nacional de Atenção Primária dispõe os deveres dos serviços básicos de atenção à saúde e, entre eles, a resolutividade. Nesse sentido, essa diretriz garante que esse nível de atenção deve ser capaz de satisfazer a maioria das necessidades de saúde da população, usando as tecnologias apropriadas de forma oportuna e eficaz. $^{1}$

O modelo de atenção à saúde, proposto pela Estratégia Saúde da Família (ESF), tem como base a instituição de um sistema centrado na atenção primária com atividades $\mathrm{e}$ serviços voltados para a promoção e prevenção da saúde, riscos e agravos, além de cura e reabilitação. Assim, as práticas educativas são consideradas uma importante linha de ação e a participação da comunidade, não apenas para a consolidação da ESF, mas do próprio SUS. ${ }^{2}$

Diante das constantes reorganizaçôes dos serviços de saúde, a ESF vem se consolidando como eixo reestruturante da atenção primária, colaborando para a produção do cuidado a partir do estabelecimento do vínculo entre usuários e equipe de saúde, escuta qualificada e participação do usuário no planejamento e nas intervenções das ações realizadas, apropriando-se do uso de tecnologias em saúde que contribuam para a sua autonomia. ${ }^{3}$

Nesse sentido, a atuação do enfermeiro na Atenção Básica vai além das ações assistenciais, pois a educação torna-se essencial no seu processo de trabalho, principalmente nas ações de promoção e prevenção em saúde. A educação em saúde engloba toda a ação do enfermeiro, seja com a equipe de saúde ou com o usuário, não sendo possível dissociar a prática assistencial, gerencial e educativa desse profissional, pois em todas as ações, o enfermeiro é considerado um educador. ${ }^{4}$
A educação

em saúde é

descrita entre as

macroprioridades

do Pacto em

Defesa da Vida.

Neste documento,

destaca-se a sua

relevância como

uma estratégia de

promoção da saúde.

Assim, trata-se de

um recurso que

oferece subsídios

para a adoção de

novos hábitos e

condutas em saúde.
Está disposta na lei do exercício profissional do enfermeiro, regulamentada em seu art.11, que cabe ao enfermeiro, como integrante da equipe de saúde realizar educação em saúde com o objetivo de melhoria da saúde do indivíduo, da família e da população em geral. Ressalta-se que essa atribuição também se encontra disposta nos objetivos da ESF.

A educação em saúde é descrita entre as macroprioridades do Pacto em Defesa da Vida. Neste documento, destaca-se a sua relevância como uma estratégia de promoção da saúde. Assim, trata-se de um recurso que oferece subsídios para a adoção de novos hábitos e condutas em saúde. As ações educativas em saúde são consideradas, em todos os níveis de atenção, um instrumento de grande relevância para promoção $\mathrm{e}$ prevenção da saúde. Na ESF isso fica bastante evidente, já que neste espaço busca-se o fortalecimento e embasamento de ações para melhoria da qualidade de vida dos usuários. ${ }^{67}$

A Educação Permanente em Saúde (EPS) é uma ferramenta propostas pelo Ministério da Saúde e pela Organização Mundial da Saúde (OMS) para a qualificação dos profissionais envolvidos na assistência a saúde. A EPS tem como foco a aprendizagem-trabalho produzida no cotidiano dos serviços de saúde, nesse processo, aprender e ensinar são incorporados ao trabalho para diminuir as diferenças entre a formação profissional e a realidade da prática do serviço de saúde'.

Diante do exposto, o presente estudo teve como objetivo compreender o protagonismo do enfermeiro nas ações de educação em saúde na Estratégia Saúde da Família.

\section{MÉTODO}

Estudo descritivo de abordagem qualitativa. Foram preservados os preceitos éticos estabelecidos pela Resolução 510/16 do Conselho Nacional de Saúde. A pesquisa 
foi aprovada pelo Comitê de Ética em Pesquisa com Seres Humanos da Faculdade Pernambucana de Saúde, com parecer número 3.033.526.

Trata-se de uma pesquisa de natureza qualitativa, que proporciona um modelo de entendimento profundo de ligações entre elementos, direcionado à compreensão da manifestação do objeto de estudo?. A população do estudo foi composta por enfermeiros de ambos os sexos, com vínculo empregatício temporário ou efetivo com a unidade de saúde há mais de um ano na ESF, possibilitando um tempo mínimo para que o profissional pudesse ter conhecimento da área adscrita ao seu território

A coleta dos dados foi realizada entre os meses de novembro de 2018 e abril de 2019, através de entrevistas semiestruturadas. Participaram do estudo 08 enfermeiros que atuavam nas ESF do município, o tamanho da amostra foi determinado pelo critério de saturação de conteúdo. Critérios de inclusão: profissionais enfermeiros com vínculo empregatício há mais de um ano na unidade de saúde, está presente na ESF no dia da coleta de dados e assinar o Termo de Consentimento Livre e Esclarecido
-TCLE. Para a análise das entrevistas, foi utilizada a técnica Análise de conteúdo na modalidade análise temática. As entrevistas foram realizadas individualmente na instituição de saúde, após a assinatura do Termo de Consentimento Livre e Esclarecido.

Neste estudo foram preservados os preceitos éticos estabelecidos pela Resolução 510/16 do Conselho Nacional de Saúde.10 A coleta dos dados só teve início após aprovação do projeto de pesquisa pelo Comitê de Ética em Pesquisa com Seres Humanos da Faculdade Pernambucana de Saúde (CEP/FPS) através do CAAE número 02317418.0.0000.5569 e Parecer número 3.033.526.

$\mathrm{O}$ instrumento utilizado para a coleta de dados foi uma entrevista semidirigida, organizada a partir de um roteiro previamente elaborado composto de perguntas abertas que possibilitassem abrir espaço para a elaboração discursiva dos próprios entrevistados. Os dados sociodemográficos dos participantes foram coletados, para uma compreensão mais aprofundada sobre o perfil da população estudada. As entrevistas foram realizadas individualmente no consultório de Enfermagem da instituição de saúde, com horário previamente acordado com cada entrevistado.

Quando da entrega do TCLE, as pesquisadoras informaram os objetivos $\mathrm{da}$ pesquisa e esclareceram eventuais dúvidas dos participantes, informando também que as entrevistas seriam audiogravadas, preservando o sigilo e anonimato dos mesmos. Para isso, no decorrer da apresentação, os entrevistados serão denominados por Enf01; Enf02; e assim por diante. Os dados foram analisados através da técnica de Análise de Conteúdo Temática proposta por Minayo, onde através dela é possível encontrar respostas para as questões formuladas e confirmar ou não as afirmações estabelecidas antes do trabalho de investigação (hipóteses). Além disso, outra função diz respeito à descoberta do que está por trás dos conteúdos manifestos, indo além das aparências do que está sendo comunicado. ${ }^{11}$

\section{RESULTADOS}

Foram entrevistados oito enfermeiros da ESF, dos quais seis eram do sexo feminino. A idade dos participantes variou entre 28 e 52 anos, cuja média de idade foi de 38,3

Tabela1- referente aos dados sociodemográficos dos participantes da pesquisa

\begin{tabular}{|c|c|c|c|c|c|c|c|c|}
\hline PARTICIPANTE & SEXO & ESTADO CIVIL & IDADE & RELIGIÃO & $\begin{array}{c}\text { TITULAÇÃO ACADÊ- } \\
\text { MICA }\end{array}$ & $\begin{array}{l}\text { TEMPO DE } \\
\text { FORMAÇÃO }\end{array}$ & $\begin{array}{l}\text { TIPO DE } \\
\text { VÍNCULO }\end{array}$ & $\begin{array}{c}\text { TEMPO DE } \\
\text { ATUAÇÃO NA ESF }\end{array}$ \\
\hline ENF 1 & $F$ & Solteira & $42 a$ & Católica & Mestrado & 15 anos & Efetivo & $>5$ anos \\
\hline ENF 2 & $\mathrm{~F}$ & Solteira & $37 a$ & Católica & Especialista & 5 anos & $\begin{array}{c}\text { Contrato } \\
\text { Tempo- } \\
\text { rário }\end{array}$ & $>5$ anos \\
\hline ENF 3 & $\mathrm{~F}$ & Casada & $28 a$ & Católica & Especialista & 3 anos & $\begin{array}{c}\text { Contrato } \\
\text { Tempo- } \\
\text { rário }\end{array}$ & 1 ano \\
\hline ENF 4 & $\mathrm{~F}$ & Solteira & $34 a$ & $\begin{array}{l}\text { Protes- } \\
\text { tante }\end{array}$ & Especialista & 10 anos & Efetivo & $>5$ anos \\
\hline ENF 5 & $\mathrm{~F}$ & Casada & 41a & $\begin{array}{l}\text { Protes- } \\
\text { tante }\end{array}$ & Mestrado & 12 anos & Efetivo & $>5$ anos \\
\hline ENF 6 & $\mathrm{~F}$ & Casada & $42 a$ & $\begin{array}{l}\text { Protes- } \\
\text { tante }\end{array}$ & Especialista & 15 anos & Efetivo & $>5$ anos \\
\hline ENF 7 & $\mathrm{M}$ & Casado & $52 \mathrm{a}$ & Católica & Especialista & 28 anos & Efetivo & $>5$ anos \\
\hline ENF 8 & $M$ & Casado & & Católica & Especialista & $<5$ anos & $\begin{array}{c}\text { Contrato } \\
\text { Tempo- } \\
\text { rário }\end{array}$ & 1 ano \\
\hline
\end{tabular}




\section{artigo}

Silva, Y. L. R., Costa, J. M.

O protagonismo do enfermeiro nas ações de educação em saúde na estratégia saúde da familia

anos. A maioria eram casados. Quanto ao tempo de formação, a maioria possuía mais de dez anos. Todos os enfermeiros possuíam algum tipo de especialização latu senso em diversas áreas da saúde e somente três na área de saúde pública. Ressalta-se que dois enfermeiros possuíam titulação de mestrado na área de Educação. Em relação às condições de trabalho, cinco eram efetivos e três eram contratados temporários. Quanto ao tempo de atuação na ESF, cinco profissionais estavam há mais de cinco anos na Unidade de Saúde e os demais atuavam há menos de 3 anos.Todos os participantes afirmaram possuir experiência em outro nível de atenção à saúde, além de afirmarem ser cristãos.

\section{Discussão}

As rodas de conversas nas ações de educação em saúde

$\mathrm{Na}$ primeira categoria os enfermeiros relataram as potencialidades e ações exitosas em suas ações educativas de saúde, destacando a promoção de ações com resultados positivos na comunidade com a participação dos Agentes Comunitários de Saúde (ACS) e a utilização de estratégias como a roda de conversa que insere o usuário como participante ativo no cuidado de sua saúde.

"As nossas atividades são feitas aqui mesmo na unidade, com a comunidade, através de rodas de conversa, de grupos." (Enf01, 42 anos).

"A gente combina com um ACS e vai pra área dele nesse dia, vão todos, eu vou também. Eu planejo com eles um tema, ai fazemos uma roda de conversa, levamos algum material impresso ou algum banner e lá fazemos... explicamos. É proveitoso." (Enf02, 37 anos)

"Usamos a roda de conversa, porque a gente acha muito importante, pois envolve a população e nesse sistema a gente vê que o êxito é melhor que as palestras." (Enf07, 52 anos)
Uma pesquisa

realizada com oito enfermeiros da ESF realizado no estado

da Paraíba, que

teve como objetivo

analisar a percepção

dos enfermeiros

em relação às suas

práticas educativas

demonstrou que

mesmo diante

das dificuldades

e desafios, pode-

se inferir que a

ESF é um cenário

que facilita ações

intersetoriais.
Os recortes das falas dos enfermeiros trazidos na presente pesquisa estão em consonância com o que foi encontrado em outros estudos já realizados. Uma pesquisa realizada com oito enfermeiros da ESF realizado no estado da Paraíba, que teve como objetivo analisar a percepção dos enfermeiros em relação às suas práticas educativas demonstrou que mesmo diante das dificuldades e desafios, pode-se inferir que a ESF é um cenário que facilita ações intersetoriais.

Não obstante, torna-se necessário que os profissionais tenham um novo olhar diante dos entraves e das ações de promoção da saúde para assim, construir novos saberes. Em adição a isso, estudo realizado com enfermeiros da ESF no estado do Ceará, identificou a importância da utilização dos espaços públicos dentro da comunidade para realização das atividades educativas. ${ }^{12-13}$

Entraves na execução das ações de educação em saúde

$\mathrm{Na}$ segunda categoria os enfermeiros elencaram as fragilidades encontradas na execução de suas práticas educativas na ESF. A carência de espaço físico, materiais e insumos foram apontados como as principais dificuldades enfrentadas no cotidiano dos profissionais enfermeiros.

"As principais são: o ambiente físico, que eu não tenho muito espaço. Às vezes, eu vou aqui pra essa área de trás que pega sol a maior parte, minhas salas são muito pequenas, às vezes está tendo atendimento". (Enf03, 28 anos)

"Falta de material, com certeza, é um dos grandes entraves. Falta recursos também para a gente modificar e sair um pouco dessa mesmice de palestra". (Enf 06, 42 anos)

Estudo realizado no Paraná ${ }^{11}$, como objetivo de identificar as dificuldades e perspectivas vivenciadas pelos enfermeiros da ESF nas ações educativas, encontrou em seus resultados diversos desafios enfrentados pelos enfermeiros na execução das ações educativas, dentre eles: a insuficiência 
de recursos físicos, materiais e financeiros, o que corrobora com os achados da presente pesquisa. Os autores apontaram, ainda, que o profissional deve buscar alternativas para realização e priorização das ações de educação em saúde como prática necessária para reorientação da Atenção Primária em Saúde. ${ }^{14}$

\section{Lacuna na educação permanente dos enfermeiros da ESF}

A educação permanente é considerada elemento essencial para o embasamento teórico do profissional de saúde. Tal fato permite o encorajamento para realização das suas práticas de forma efetiva, impactando na saúde dos usuários. A falta de investimento em educação permanente por foi apontada pelos participantes como algo negativo, conforme pode ser observado nos relatos a seguir:

"Como é uma coisa que eu nunca fui capacitado para isso, então eu não tenho interesse de fazer algo que eu não sei abordar". (Enf08, 28 anos)

"Porque assim é muito complicado. Eu chegar aqui e jogar todo mundo numa recepção e começar a falar $e$ tirar foto é muito prático. Mas eu quero saber se aquela população entendeu, se eu vou impactar aquela pessoa...que transforme comportamento, que entenda a necessidade da mudança". (Enf04, 34 anos)

"Capacitação, principalmente para você lidar com a dinâmica de uma roda de conversa". (Enf05, 41 anos)

A Educação Permanente em Saúde tem como objetivos principais o de gerar reflexão sobre o processo de trabalho, autogestão, mudança institucional e transformação das práticas profissionais. ${ }^{15}$

Estudo realizado em Goiânia-GO, com o objetivo verificar o significado e contribuições da educação permanente sob a ótica dos enfermeiros que atuam na Estratégia Saúde da Família, constatou que a educação permanente permite ao profissional capacitado planejar, organizar, desenvolver e avaliar ações que atendam às necessidades daquela população. Para isso, faz-se necessário uma interação contínua com os usuários, com a finalidade de mobilizá-los e estimular a participação deles nesse processo. ${ }^{16} \mathrm{Os}$ dados deste estudo também coincidem com as falas dos enfermeiros que participaram desta pesquisa no sertão de Pernambuco, à medida que os entrevistados sinalizaram que a falta de capacitação gera insegurança para pensar em medidas mais inovadoras.

\section{CONCLUSÕES}

Os resultados do estudo apontam que, mesmo diante dos desafios e fragilidades, os enfermeiros realizam atividades que envolvem os usuários de forma ativa do processo educativo, principalmente, através de rodas de conversa e ações dentro dos espaços públicos da comunidade.

A educação em saúde surge como uma estratégia reorientadora do cuidado, o que reflete na melhoria dos indicadores de saúde da população. Assim, para que tal objetivo seja alcançado é importante o investimento na educação continuada e o incentivo por parte dos gestores públicos para que as ações educativas em saúde possam ser exitosas e os profissionais desenvolvam um novo olhar diante dos usuários, valorizando seus saberes e compreendendo o contexto social e cultural em que estão inseridos.

Ressalta-se que os dados encontrados não podem ser generalizados, uma vez que o estudo foi realizado com uma população específica de um lugar específico. Desta maneira, recomenda-se outros desenhos de estudo dentro da abordagem qualitativa e mista visando acrescer ainda mais luz à complexidade do fenômeno.

\section{REFERÊNCIAS}

1. Cookson R, Mondor L, Asaria M, Kringos DS, Klazinga NS, Wodchis WP. Primary care and health inequality: Difference-in-difference study comparing England and Ontario. PLOS ONE.2017; 12(11)

2.Oliveira LC, Ávila MMM, Gomes AMA, Sampaio MHLM. Popular participation in health education initiatives: challenges for primary healthcare professionals. Interface (Botucatu).2014; 18 Supl 2:1389-1400

3. Silocchi C, e Junges JR. Equipes de atenção primária: dificuldades no cuidado de pessoas com doenças crônicas não transmissíveis. Trab. Educ. Saúde, 2017;15( 2): 599615

4. Lubini, V.T.; Willrich, J.Q.; Pickersgill, M.F.; Portela, D.L. A educação em saúde como estratégia de cuidado de enfermagem na atenção básica. saúde coletiva 2019; 47 (09): 5. Roecker S, Nunes EFPA, Marcon SS. 0 trabalho educativo do enfermeiro na estratégia saúde da familia. Texto Contexto Enferm, Florianópolis, 2013; 22(1): 157-65.

6. Ministério da Saúde (Br). Política Nacional de Promoção da Saúde. Brasilia: Ministério da Saúde; 2010

7. Andrade ACV, Schwalm MT, Ceretta LB, Dagostin VS, SorattoO MT. Planejamento das ações educativas pela equipe multiprofissional da Estratégia Saúde da Familia. Mundo da Saúde, São Paulo - 2013;37(4):439-449.

8. Oliveira, A.M.F.; Lourenço, M.P.; Labegalini, C.M.G.; Benedetti, G.M.S.; Soares, J.P.R.; Costa, M.A.R.Educação interprofissional na atenção primária à saúde: perspectivas e vivências. saúdecoletiva $\cdot 2021 ; 64(11)$.
9. Minayo, MC. O desafio do conhecimento: pesquisa qualitativa em saúde. São Paulo, 2007.

10.BRASIL. Conselho Nacional de Saúde. Resolução n 510, de 7 de abril de 2016. Diário Oficial da União:Seção 1. p. 44-46.

11. Fontanella BJ. Ricas I \&Turato ER. Amostragem por saturação em pesquisas qualitativas em saúde: contribuições teóricas. Cad. Saúde Pública, 2008; 24(1): 17-27.

12. Oliveira MB, Cavalcante EGR, Oliveira DR, Leite CEA, Machado MFAS.Educação em saúde como prática de enfermeiros na estratégia saúde da familia. Rev Rene.201314(5):894-903.

13. Trigueiro JS, Silva ACO, Góis GAS, Almeida SA, Nogueira JA, Sá LD.Percepção de enfermeiros na educacão em saúde na tuberculose Cienc Cuid Saude 2009 Out/Dez: 8(4):660-666. http://dx.doi.org/ 10.4025/cienccuidsaude.v8i4.9697.

14. Roecker S, Budó MLD, Marcon SS .Trabalho educativo do enfermeiro na Estratégia Saúde da Família: dificuldades e perspectivas de mudanças.Rev Esc Enferm USP [Internet] 2012 [citado 2019 jul.7]; 46(3):641-9.

15. Ministério da Saúde(BR), Política Nacional de Educação Permanente em Saúde: o que se tem produzido para o seu fortalecimento? / Diário Oficial da União: República Federativa do Brasil; 2018. Jul 13, Seção 1: p.59.

16. Paulino VCP, Bezerra ALQ, Branquinho NCSS, Paranaguá TTB. Educação permanente e saúde da familia. Rev. enferm. UERJ, Rio de Janeiro, 2012 jul/set; 20(3):312-6 2012. http://dx.doi.org/10.4025/cienccuidsaude.v8i4.9697. 